\title{
Biochip With Multi-Planar Electrodes Geometry for Differentiation of Non-Spherical Bioparticles in a Microchannel
}

\author{
Amina Farooq \\ Lahore University of Management Sciences \\ Nauman Butt \\ Lahore University of Management Sciences \\ Umer Hassan ( $\nabla$ umer.hassan@rutgers.edu ) \\ Rutgers, The State University of New Jersey
}

\section{Research Article}

Keywords: Multi-planar, Electrodes, cells, microchannel

Posted Date: February 17th, 2021

DOI: https://doi.org/10.21203/rs.3.rs-199679/v1

License: (c) (i) This work is licensed under a Creative Commons Attribution 4.0 International License.

Read Full License 


\section{Biochip with Multi-planar Electrodes Geometry for Differentiation of Non- Spherical Bioparticles in a Microchannel}

Authors: Amina Farooq ${ }^{1,2}$, Nauman Z. Butt ${ }^{2}$, Umer Hassan*1,3

${ }^{1}$ Department of Electrical and Computer Engineering, School of Engineering, Rutgers The State University of New Jersey, Piscataway, NJ, USA

${ }^{2}$ Department of Electrical Engineering, Lahore University of Management Sciences, Lahore. Pakistan

${ }^{3}$ Global Health Institute, Rutgers The State University of New Jersey, New Brunswick, NJ, USA

*Corresponding Author: umer.hassan@rutgers.edu; Tel (848) 445-2164 


\begin{abstract}
A biosensor capable of differentiating cells or other microparticles based on morphology finds significant biomedical applications. Examples may include morphological determination in the cellular division process, differentiation of bacterial cells, and cellular morphological variation in inflammation and cancer etc. Here, we present a novel integrated multi-planar microelectrodes geometry design that can distinguish a non-spherical individual particle flowing along a microchannel based on its electrical signature. We simulated multi-planar electrodes design in COMSOL Multiphysics and have shown that the changes in electrical field intensity corresponding to multiple particle morphologies can be distinguished. Our initial investigation has shown that top-bottom electrodes configuration produces significantly enhanced signal strength for a spherical particle as compared to co-planar configuration. Next, we integrated the co-planar and top-bottom configurations to develop a multi-planar microelectrode design capable of electrical impedance measurement at different spatial planes inside a microchannel by collecting multiple output signatures. We tested our integrated multi-planar electrode design with particles of different elliptical morphologies by gradually changing spherical particle dimensions to the non-spherical. The computed electrical signal ratio of non-spherical to spherical particle shows a very good correlation to predict the particle morphology. The biochip sensitivity is also found be independent of orientation of the particle flowing in the microchannel. Our integrated design will help develop the technology that will allow morphological analysis of various bioparticles in microfluidic channel in the future.
\end{abstract}




\section{Introduction}

Single-cell analysis is critically important in biological and clinical research for personalized disease diagnostics and therapeutics ${ }^{1,2}$. Characterization of individual cells offers detailed insights into their pathological condition and are therefore of huge interest to medical specialists ${ }^{3}$. Such data empowers a reliable diagnosis of diseases, infections, and biological threats ${ }^{4}$. Of the different strategies proposed recently for single-cell analysis, cell impedance spectroscopy has pulled specific consideration because of the insights it provides into the mechanical, physical and biochemical properties of biological cells. Single cell impendence analysis helps medical experts with the methods not exclusively to identify the advancement of a disease during its very earliest phase, but also adequacy of treatment strategies ${ }^{5,6}$. Currently, there are several means of measurement that are typically used to detect cell by their size or volume. Importantly, the impedimetric cytometer based on coulter principle detects differential electrical signal of a biological cell under the influence of an externally applied electrical field ${ }^{7-10}$. This has many applications such as separating diseased cells from healthy cells, platelets from the whole blood, live from dead cells, budding yeast cells classification, and, contaminants from the pure water, etc. Among various platforms, microfluidic impedimetric cytometry is established and frequently used in several medical applications due to low cost, label-free, high precision, and evident integration with on-chip electronic circuit for portable biosensors ${ }^{11-13}$.

The latest research highlights numerous numerical and exploratory examinations concerning different aspects of single-cell impedance spectroscopy such as cell morphology differentiation along with usual parameters such as size, volume, viability, and opacity ${ }^{14-19}$. Biological cells have variable shape and size like rod-shaped bacteria and biconcave shaped RBCs. Separation of nonspherical bioparticle is challenging because all existing single-cell methods rely upon the detection 
criteria based on the size and/or volume of a bioparticle i.e. diameter. Cell shape differentiation is a key physiological cellular condition aids in medical analysis and microorganism growth parameter monitoring. For example, separation of live cells from dead cells through differences in electrical (i.e., conductivity and permittivity) and mechanical (i.e., size and shape) properties of cells ${ }^{16}$. Sickle cell anemia erythrocytes are rigid sickle-shaped RBCs due to abnormal hemoglobin polymerization which leads to cell rigidity and vasocculation ${ }^{20}$. Y. Katsumoto et al. presented a sensor which can differentiate normal human RBCs from glutaraldehyde-treated (rigidified) as they were stretched by the high shear flow in a microchannel ${ }^{2}$. K. Asami designed spherical and elliptical shell models for investigating bacterial and yeast cell growth in cultures ${ }^{1}$. Kotb MA et al. successfully differentiated the normal human RBCs from the benzene exposed worker cells even at a very low level of exposure ${ }^{21}$. Malaria infection causes swelling of the erythrocyte with shape change from biconcave to spherical related to degradation of the cytoskeleton due to invasion of the parasite; studied in detail by Alan F. Cowman and B. S. Crabb ${ }^{22}$. Ting Ye et al. develops two types of cell model for investigating the stretching deformation shape relaxation time for malaria infected $\mathrm{RBCs}^{17}$. The infected cells gradually lose their deformability and their ability to recover their original shape is greatly reduced with the maturation of the parasites. It is a complex dynamic process, accompanied by progressive changes in the shape, size, and mechanical properties of the cell. Furthermore, circulating tumor cells (CTCs) also exhibit highly variable shapes, including round, oval, elongated, and clusters. Non-round and multi-nucleate cells were sometimes observed $^{14,23}$. Differentiation is based on large cancer cell size (e.g. Prostate cancer cell size ranged from $6.9-8.95 \mu \mathrm{m}^{14}$ and lung tumor cells ${ }^{15}$ ). Similarly, CTCs derived from breast cancer have a median cell diameter of $13.1 \mu \mathrm{m}^{24}$, and size-based method to isolate CTCs rely on their increased $\operatorname{size}^{25}$. B. Shashni et al. highlighted difference in surface deformity (roughness) between cancer 
cell lines (SP2/O cells and LLC cells) and normal cells (WBC and lymph node cell) ${ }^{26}$. Cell shape variation is predominant at some point in cell division or mitosis. Heterogeneous populations of budding yeasts have been analyzed and spherical, early-stage element and grown-up yeast were distinguished 27,28 . In addition, shape analysis of microorganism permits classification of microorganism in accordance with their type and might be used for microorganism anomalous growth diagnosis $^{4,29,30}$. Moreover, K. K. Zeming and S. Ranjan separated the non-spherical bioparticle by using new array design in a microfluidic device ${ }^{30}$. The COMSOL Multiphysics has been used in the past for many biosensor projects. For instance, a COMSOL simulation on a single HeLa (human cervical epithelioid carcinoma) cell was developed to determine electrical properties (impedance, conductivity, and permittivity) to analyze the different cellular processes using a broad selection of voltage and frequency preconditions ${ }^{3}$. Sheng Hu et al. discussed in detail single cell dielectrophoretic (DEP) behavior in optoelectronic tweezers based on COMSOL ALE simulations ${ }^{31}$. B. Brazey et al. presented a comparison of two electrode geometries, square and star shape, via FEM simulation in COMSOL and then develop a microfluidic device for experimental validation ${ }^{32}$.

In this paper, we designed a novel multi-planar microelectrodes system for electrical differentiation of non-spherical bioparticles as they pass through a microchannel. Multi-planar microsensor is equipped with hybrid design of co-planar and top-bottom electrodes configurations. The passage of single particle through the channel result in producing electrical pulses with distinct features associated with different morphologies. Particle morphology is varied by gradually changing the spherical axis quotient from low to high value i.e. spherical to non-spherical (ellipsoidal) transformation. We demonstrated the efficiency of the morphology detection by flowing these particles through the channel and obtaining corresponding electrical signatures. The 
novelty of our device includes demonstration of efficient differentiation of particles with different morphology producing unique electrical characteristics in a novel multi-planar electrode configuration. Our presented configuration can potentially be used to explore several particles differentiations such as pathogens, RBCs, budding yeasts with enhanced sensitivity and efficiency, evading the constraint of spherical dimension from conventional methods.

\section{Methods}

\section{Microfluidic channel with microelectrodes design}

The single-cell analysis method is modeled utilizing the COMSOL Multiphysics simulation software. To create models for single cell microfluidic device the AC/DC module of the COMSOL suite is used. Fig. 1 shows a schematic 3-D diagram of the microfluidic channel with different configurations of microelectrodes employed in this study. The channel width, $\mathrm{W}$ is $100 \mu \mathrm{m}$ and the channel height, $\mathrm{H}$ is $15 \mu \mathrm{m}$. Two sensing zone areas with dimensions of length $\times$ width $\times$ height as $30 \mu \mathrm{m} \times 15 \mu \mathrm{m} \times 15 \mu \mathrm{m}$ are modelled within the microchannel. The focused region allows the electric field lines to be more concentrations in the sensing zone, thereby producing higher signalto-noise ratio signals ${ }^{23}$. Microelectrode material is selected as Platinum with a thickness of 100 $\mathrm{nm}$. The changes in the electric field intensity with the passage of particle will be measured at respective electrodes surface.

A conventional coplanar microelectrode design (Design A) is comprised of three co-planar electrodes of $100 \mu \mathrm{m}$ width are separated by a gap of $35 \mu \mathrm{m}$ and positioned at the bottom of microchannel outside both sensing zones as illustrated in Fig. 1a. To demonstrate the efficacy of sensing in the focused zone, we shifted the periphery electrodes to the bottom of the zone as illustrated in Fig. 1b (Design B). The middle bottom platinum electrode is excited by an AC 
voltage of $10 \mathrm{~V}$ and the corresponding changes in the electric field intensity is recorded between the exterior pair of platinum electrodes $\left(E_{1}\right.$ and $\left.E_{2}\right)$. As the modeled particle sweeps through the microchannel, a differential bipolar pulse is produced whose amplitude is proportional to the diameter of the particle.

Top-bottom electrodes configuration design (Design C) is shown in Fig. 1c, where microelectrodes are placed exactly at the top and bottom planes of the sensing zones. The electrical field inside the microchannel is governed by the Laplace equation,

$$
\Delta V=0
$$

with the insulating boundary conditions at the channel walls and reservoirs, and with specified electrical potentials at the electrodes. The voltage potential is $10 \mathrm{~V}$, and the functional form of the transient electrical field is the sine wave in this study. Since the Reynolds number is low, inertia terms can be neglected and the flow field inside the microchannel can be governed by the Stokes equation as, with the no-slip boundary conditions at the channel walls and with specified pressure values at the reservoirs. The input voltage signal is applied to the bottom electrodes and a differential signal (electric field intensity) is obtained from the top electrodes.

\section{Electric field intensity measurements}

The electric conductivity of a particle is generally a value small as compared to the medium in which particle is suspended e.g. PBS solution. The resulting resistance between the electrodes increase considerably once a particle passes between them in the microchannel flow because of their electrical properties. Similarly, electrical permittivity of the particle and medium is frequency dependent and selection of appropriate frequency to be applied to the electrodes in critically important. The frequency range of $200-500 \mathrm{kHz}$ can be used to obtain the output signature 
corresponding to the size and volume of the particle. We used a frequency of $300 \mathrm{kHz}$ to electrically categorize particles based on their size and shape depending on the degree of difference between the resistances of the fluid properties and the particle membrane electrical parameters (conductivity and relative permittivity). The changes in the electric field intensity are measured at two sets of electrodes and a differential signal is proposed to minimize the common mode gain (a common cause of noise in electrical measurements). We found that the profile of $\Delta \mathrm{E}$ signal (differential bipolar pulse) e.g., maximum $\Delta \mathrm{E}$ peak, is affected not only by the size of the particle but also by its shape, position and the physical properties of the particle and the fluid properties. In this study, the position of the particle is fixed at the center of the detection volume and we sweep the particle along $\mathrm{x}$-axis through both sensing regions of the microfluidic channel for measurements. We evaluate the shape and size of the particles by analyzing the profile of the $\Delta \mathrm{E}$ signal as the particle passes through the sensing regions.

\section{COMSOL material specifications}

Following material properties were used in selection of microparticles, fluid in the microchannel and electrode material. Biological cell/ Microparticle: Microparticle is modelled as a biological cell with material specifications of 50 relative permittivity $\left(\varepsilon_{\mathrm{r}}\right), 0.67 \mathrm{~S} / \mathrm{m}$ electrical conductivity, $9 \times 10^{-4} \mathrm{~K}^{-1}$ coefficient for thermal expansion $(\alpha), 1460 \mathrm{~J} /(\mathrm{kg}-\mathrm{K})$ heat capacity at constant pressure $(\mathrm{Cp}), 970 \mathrm{~kg} / \mathrm{m}^{3}$ density $(\rho), 0.16 \mathrm{~W} /(\mathrm{m} * \mathrm{~K})$ thermal conductivity at $25^{\circ} \mathrm{C}(\mathrm{k}), 7.5 \times 10^{8} \mathrm{~Pa}$ elastic modulus (E), and 0.5 Poisson's ratio (v).

Phosphate Buffer Saline (PBS): Microchannel geometry is filled with 1x PBS solution at $\mathrm{pH}$ 7.4, which has specific material properties of 80 relative permittivity $\left(\varepsilon_{\mathrm{r}}\right), 0.88 \mathrm{cP}$ dynamic viscosity $(\mu)$ at $25{ }^{\circ} \mathrm{C}, 1.6 \mathrm{~S} / \mathrm{m}$ electrical conductivity $(\sigma), 4.186 \times 10^{3} \mathrm{~J} /(\mathrm{kg}-\mathrm{K})$ heat capacity at constant pressure (Cp), $997 \mathrm{~kg} / \mathrm{m}^{3}$ density $(\rho)$, and $0.598 \mathrm{~W} /(\mathrm{m}-\mathrm{K})$ thermal conductivity at $25^{\circ} \mathrm{C}(\mathrm{k})$. The 
material properties used for the microfluidic design modelled in COMSOL Multiphysics 5.3a (conductivity and relative permittivity) employed in all simulations within this article have been summarized in Table S1.

Microelectrodes: We choose platinum material for the electrodes. Electrode material properties are selected as, 7 relative permittivity $\left(\varepsilon_{\mathrm{r}}\right), 8.9 \times 10^{6} \mathrm{~S} / \mathrm{m}$ electrical conductivity, $8.8 \times 10^{-6} \mathrm{~K}^{-1}$ coefficient for thermal expansion $(\alpha), 133 \mathrm{~J} /(\mathrm{kg}-\mathrm{K})$ heat capacity at constant pressure $(\mathrm{Cp})$, $2.15 \times 10^{4} \mathrm{~kg} / \mathrm{m}^{3}$ density $(\rho), 71.6 \mathrm{~W} /(\mathrm{m}-\mathrm{K})$ thermal conductivity at $25^{\circ} \mathrm{C}(\mathrm{k}), 1.68 \times 10^{2} \mathrm{MPa}$ elastic modulus (E), and 0.38 Poisson's ratio (v). In COMSOL electrostatics module, PBS filled the microchannel geometry was designated with zero charge and assigned with an initial value of $0 \mathrm{~V}$. Charge was conserved through the constitutive relationship between electric field strength and relative permittivity:

$$
\mathrm{E}=\mathrm{D} / \varepsilon_{0} \varepsilon_{\mathrm{r}}
$$

where $\mathrm{E}$ is the electric field, $\mathrm{D}$ is dielectric constant, $\varepsilon_{\mathrm{r}}$ is relative permittivity, and $\varepsilon_{0}$ is the relative permittivity of free space.

Mesh size: In our 3-D simulations, free tetrahedral meshing was used with 'finer' settings in COMSOL. Maximum element size for this setting is $2.86 \mu \mathrm{m}$, minimum element size of $0.25 \mu \mathrm{m}$ and element growth rate of 1.45 .

\section{Results and Discussion}

\section{Multiplex configurations of microelectrodes}

Two most employed microelectrode configurations in the impedimetric microfluidic cytometer are

coplanar and top-bottom electrode geometries ${ }^{33-43}$ In the conventional coplanar electrode design 
pair of electrodes have been placed at the bottom of microfluidic channel besides both sensing regions defined in microchannel. The electric potential has been applied to the middle bottom electrode at the center. When a particle passes between the set of microelectrodes through the sensing region, the change in differential electrical field distribution $\left(E_{1}-E_{2}\right)$ has been recorded from the exterior electrodes as a bipolar pulse i.e. Design A (Fig. 1a). We modified this coplanar microelectrode configuration by shifting the exterior microelectrodes to the bottom of both focused sensing regions while keeping the middle electrode at the same position i.e. Design B (Fig. 1b). For top-bottom electrode configuration the electrodes are aligned at top and bottom parts of the microchannel at the focused regions i.e. Design C. The input signal is applied to pair of bottom electrodes and the differential electrical field data is obtained from top electrodes pair (Fig. 1c). Coplanar electrode configuration is prevalent due to its ease of fabrication; however, top-bottom electrode layout provides higher sensitivity due to uniform electric field distribution between electrodes across vertical axis of microfluidic channel.

We compared the performance of these electrode configurations by flowing a spherical particle with a diameter of $10 \mu \mathrm{m}$ with electrical conductivity, $\sigma=0.67 \mathrm{~S} / \mathrm{m}$, and relative permittivity, $\varepsilon=50$. The bioparticle is swept through all three design layouts (Design A, B, \& C) in COMSOL Multiphysics to yield the electrical signature for the spherical bioparticle. The resulting differential electrical field signals $\left(E_{1}-E_{2}\right)$ for all design layouts are shown in Fig. 1d. A single bioparticle passing through the Design A layout can be seen in the inset of Fig. 1d. The signal strengths for the Design A, B, C configurations are $6.94 \mathrm{kV} / \mathrm{m}, 147.5 \mathrm{kV} / \mathrm{m}$ and $184.2 \mathrm{kV} / \mathrm{m}$ respectively. The standard deviation of the noise $(\sigma)$ for the Design A, B, C configurations are $0.7 \mathrm{kV} / \mathrm{m}, 8.7 \mathrm{kV} / \mathrm{m}$ and $1.3 \mathrm{kV} / \mathrm{m}$ respectively. Design A produced much smaller signal strength as compared to Designs B and C, which is also evident by Signal-to-noise (SNR) values shown in Supplementary 
Table 2. Design $\mathrm{C}$ performed the best with highest signal strength and lowest noise. The modification in existing coplanar electrode layout has proved to be substantially sensitive with enough SNR enabling better detection of $10 \mu \mathrm{m}$ diameter particles. The peak signal strength values and the corresponding SNR values for all three design configurations are shown in Fig. 1d. In addition, we also straighten the center space in Design C as shown in Supplementary Figure S1. We performed the comparative analysis and flowed a $10 \mu \mathrm{m}$ biological particle $(\sigma=0.67 \mathrm{~S} / \mathrm{m}, \varepsilon=$ 50) across the microchannel between the electrodes. The results are show in Figure $\mathbf{S 2}$ and Table S3. Design C with broad center space resulted in higher signal strength, lower noise and higher SNR.
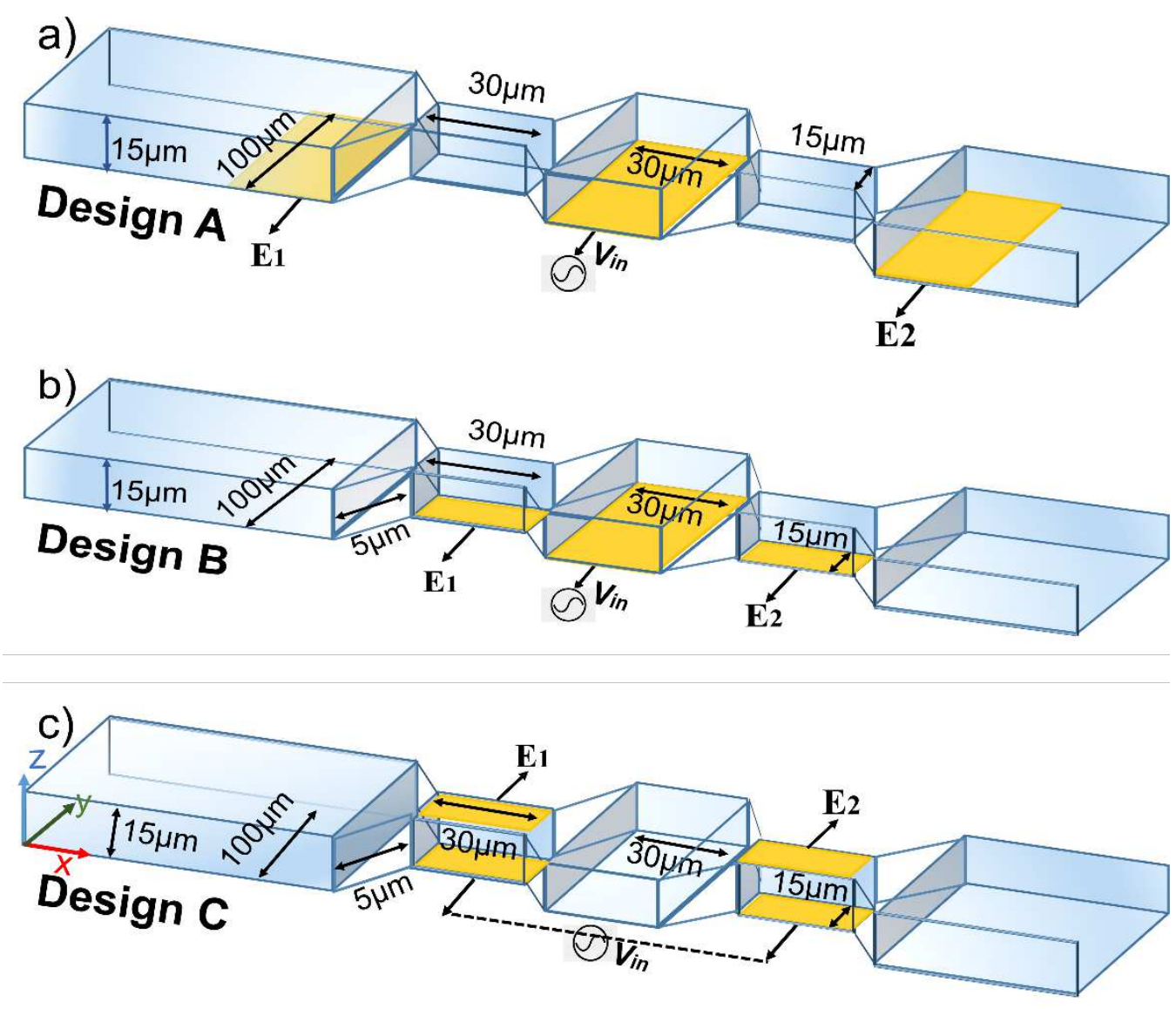

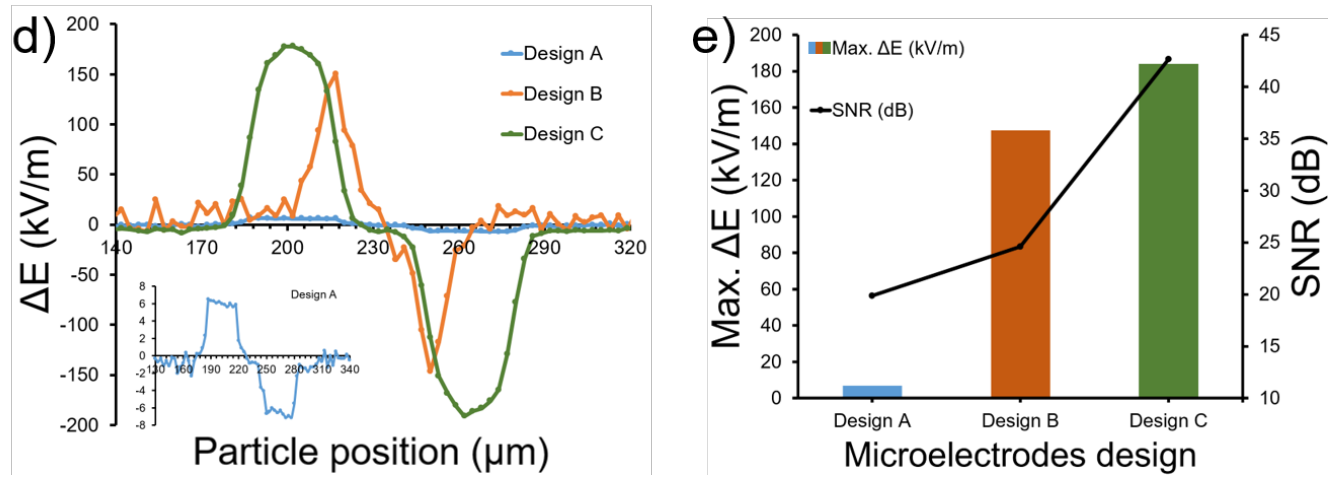

Figure 1. 3-D view of all microfluidic channels with microelectrodes designs with geometrical dimensions, a) Coplanar microelectrodes layout (Design A), b) Modified coplanar microelectrode layout (Design B) with electrodes in narrow aperture, c) Top bottom microelectrodes layout (Design C), d \& e) Electrical signal comparison in three microelectrode layouts. Design B, C outperformed Design A in signal strength. However, Design C produces highest signal strength and lowest noise.

\section{Integrated multi-planar configuration of microelectrodes (IE)}

The profiles of electric field lines for co-planar and top-bottom design configurations are shown in Supplementary Figure 3. Design C shows the uniform electric field lines across the vertical height. However, for Design $\mathrm{B}$, the electric field lines are originating from middle to side electrodes in horizontal direction. We propose to combine the Design B and C configurations to develop a novel integrated multi-planar microelectrode configuration (IE) capable of morphological single cell analysis. The IE configuration was designed by integrating top-bottom and coplanar electrode layouts, allowing to perform two-fold single bioparticle sensing. We implemented IE configuration with two possible signal excitation designs which are shown in Fig.

2. The effect of grounding and applied signal to the electrodes pair were reversed and analyzed as illustrated in 3-D view of both designs. In IE-1 excitation signal is provided to bottom electrodes and multiple output signals are collected as $\Delta \mathrm{E}=\mathrm{E}_{1}-\mathrm{E}_{3}$ and $\mathrm{E}_{2}$, where $\Delta \mathrm{E}$ is a differential bipolar signal and $E_{2}$ is a non-differential unipolar signal (Fig. 2a). In IE-2 excitation signal is provided 
to top electrodes and multiple output signals are measured as $\Delta E=E_{1}-E_{3}$ and $E_{2}$ (Fig. 2b). Note that these two designs will generate a different electric field profile with respect to the middle bottom electrode. The electric field profiles are shown in Supplementary Figure 4. We performed a comparative analysis and flowed a $10 \mu \mathrm{m}$ particle across the microchannel over the electrodes. The resulting $\Delta E=E_{1}-E_{3}$ and $E_{2}$ signatures for both IE-1 and IE-2 designs are shown in Fig. 2c. In Fig. 2c and Fig. 2d we can observe that $\Delta \mathrm{E}$ signal peak values for both IE-1 and IE-2 are approximately identical, however, the noise level of $\Delta \mathrm{E}$ signal for IE-2 is much larger compared to IE-1. This results in much larger SNR for IE-1 (40.29 dB) compared to IE-2 (30.96 dB). The relevant values for noise, signal peak and SNR for $\Delta \mathrm{E}$ signals can be found in Supplementary Table S4. Similarly, E2 signal peak values for both IE-1 and IE-2 are approximately identical, however, the noise level of $E_{2}$ signal for IE-1 is much larger compared to IE-2. This results in much larger SNR for IE-2 (21.52 dB) compared to IE-1 (34.17 dB). The relevant values for noise, signal peak and SNR for $E_{2}$ signals can be found in Supplementary Table S4. These unique differences of the noise between different designs can be explained by the electric field distribution profiles. For IE1 design, there is a uniform electric field lines distribution from bottom electrode to top electrode, while there is non-uniform electric field distribution from bottom electrodes (where input signal is applied) to middle bottom electrode. Hence, we obtain $\Delta \mathrm{E}$ with better $\mathrm{SNR}$ and $\mathrm{E}_{2}$ with less $\mathrm{SNR}$. However, for IE-2 configuration, electric field vector starts from top electrodes and ends at all three bottom electrodes. This results into a more uniform electric field distribution throughout the sensing region. Therefore, it produces better SNR for both signals especially for $E_{2}$. Further, note that the difference in both signals $\left(\Delta \mathrm{E}\right.$ and $\left.\mathrm{E}_{2}\right) \mathrm{SNR}$ is much larger in IE-1 design as compared to IE-2. 

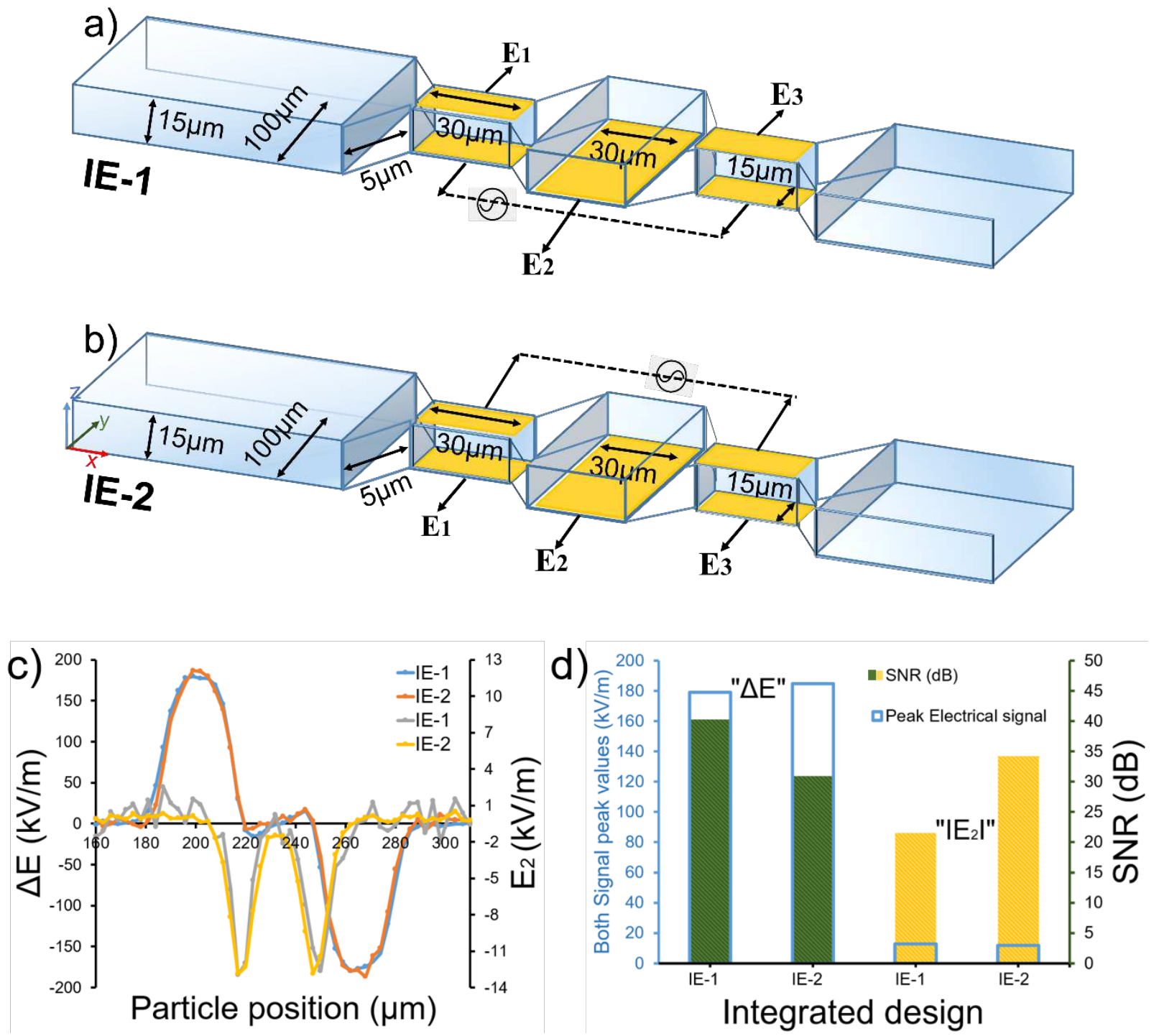

Figure 2. Detailed geometrical dimensions for integrated multi-planar designs of microelectrodes (IE-1 and IE-2) with microfluidic channel in 3-D view, a \& b) Integrated electrode designs with input signal and output signals; IE-1 (a) and IE-2 (b), c) Output signals $\Delta E=E_{1}-E_{3}$ (bipolar pulse) and $E_{2}$ (unipolar pulse) from IE-1 and IE-2 relative to the particle position in the channel, d) Comparison between the $\Delta \mathrm{E}=\mathrm{E}_{1}-\mathrm{E}_{3}$ and $\mathrm{E}_{2}$ signal peak values with respect to $\mathrm{SNR}$ in $\mathrm{dB}$ for IE-1 and IE-2.

\section{Sensitivity determination of the integrated design}

Next, we determined the sensitivity of device by simulating a spherical shape particle (modelled as a biological cell with electrical properties of $\varepsilon=50, \sigma=0.67 \mathrm{~S} / \mathrm{m})$. We analyzed the particle 
sizes (diameter) ranging from 1 to $10 \mu \mathrm{m}$. The simulation test determines the sensitivity and limit of detection (LOD) for the integrated designs when a single particle of varying size is traversed through the microchannel. Figure 3 illustrates particle of different diameters and their respective electrical signatures. Using IE-1 design configuration, $\Delta \mathrm{E}$ and $\mathrm{E}_{2}$ signatures for 1-10 $\mu \mathrm{m}$ particles are shown in Fig. 3a and Fig. 3b respectively. Fig. 3c shows exponential increase in the maximum $\Delta \mathrm{E}$ values vs. particle diameter. For $10 \mu \mathrm{m}$ particle SNR was found to be $40.2 \mathrm{~dB}$. Reduction in the signals' strength is $26 \%$ and $46 \%$ for a $9 \mu \mathrm{m}$ and $8 \mu \mathrm{m}$ respectively compared to a $10 \mu \mathrm{m}$ particle. Similarly, a $7 \mu \mathrm{m}, 6 \mu \mathrm{m}$ and $5 \mu \mathrm{m}$ particles' resulting electrical signal percentage difference decreases up to 64, 77 and 95 percent respectively. The $5 \mu \mathrm{m}$ and less diameter particles saw more than 96 percent decrease which is also visually evident from Fig. 3a. Similar analysis is also done for $\mathrm{E}_{2}$ signal and is shown in Fig. 3b, d. All the relevant values including $\Delta \mathrm{E}$, $|\mathrm{E} 2|$, noise and SNR are reported in Supplementary Table 5. The $\Delta \mathrm{E}$ signal peak (Fig. S5a) for $1 \mu \mathrm{m}$ and $2 \mu \mathrm{m}$ particles and $\mathrm{E}_{2}$ signal negative peak (Fig. $\mathbf{S 5 b}$ ) for $1-4 \mu \mathrm{m}$ particles are not distinguishable from noise. Corresponding $\Delta \mathrm{E}$ and $\mathrm{E}_{2}$ signals are shown in Supplementary Figure 5. Limit of detection can be found by setting desired SNR value to be around $10 \mathrm{~dB}$ for the $\Delta \mathrm{E}$ and $\mathrm{E}_{2}$ signature. For IE-1 design it comes to be $3 \mu \mathrm{m}$ for $\Delta \mathrm{E}$ signal and $5 \mu \mathrm{m}$ for $\mathrm{E}_{2}$. Similarly, for IE-2 design configuration, $\Delta \mathrm{E}$ and $\mathrm{E}_{2}$ signatures for 1-10 $\mu \mathrm{m}$ particles are shown in Supplementary Fig. S6a and Fig. S6b respectively. Fig. S6c shows a similar trend of exponential increase in the maximum $\Delta \mathrm{E}$ values vs. particle diameter. For $10 \mu \mathrm{m}$ particle SNR was found to be $31.1 \mathrm{~dB}$ and $34.8 \mathrm{~dB}$ for $\Delta \mathrm{E}$ and $\left|\mathrm{E}_{2}\right|$ signals respectively. Reduction in the signals' compared to a $10 \mu \mathrm{m}$ particle is shown in Fig. S4c and Fig. S6d for $\Delta \mathrm{E}$ and $\left|\mathrm{E}_{2}\right|$ signals respectively. The $4 \mu \mathrm{m}$ and less diameter particles produces much smaller signal strengths and were indistinguishable from noise. The corresponding values including $\Delta \mathrm{E},\left|\mathrm{E}_{2}\right|$, noise and SNR for IE-2 design are reported in Supplementary Table S6. The 
$\Delta \mathrm{E}$ signal peak (Fig. S7a) and $\mathrm{E}_{2}$ signal negative (Fig. S7b) for 1 to $4 \mu \mathrm{m}$ particles are not distinguishable from noise. Corresponding $\Delta \mathrm{E}$ and $\mathrm{E}_{2}$ signals are shown in Supplementary Figure S7. Note that employing subsequent signal processing techniques (noise filters) can help improve the sensitivity and detection of these smaller particles in both IE-1 and IE-2 designs. Furthermore, SNR value, limit of detection and sensor sensitivity can be further improved by reducing the channel height, increasing signal strength, and with reduced electrodes spacing. 

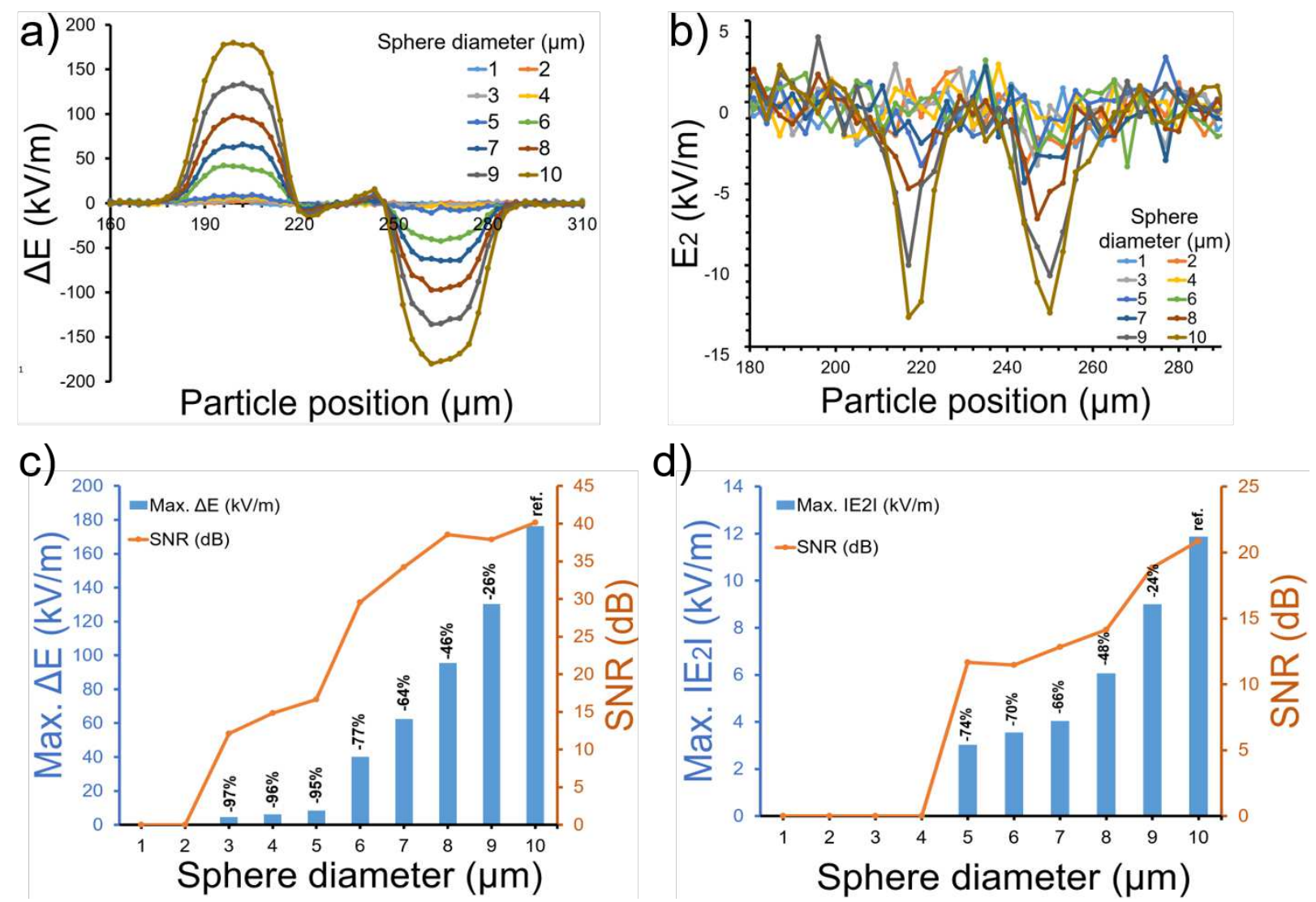

Figure 3. $\mathrm{a} \& \mathrm{~b}$ ) Simulation of $\Delta \mathrm{E}$ and $\mathrm{E}_{2}$ signals for spherical particle versus diameter increment from 1 to 10 in $\mu \mathrm{m}, \mathrm{c} \& \mathrm{~d}$ ) Comparison of maximum values of $\Delta \mathrm{E}$ and $\left|\mathrm{E}_{2}\right|$ and their resctive SNR in $\mathrm{dB}$ for spherical particle diameter ranges from 1 to $10 \mu \mathrm{m}$.

\section{Spherical to non-spherical (or ellipsoidal) bioparticle discrimination}

Biological species come in many shapes and sizes, many are non-spherical in shape; e.g. discshaped red blood cells and rod-shaped bacteria. As a first step to distinguish the non-spherical particle we focused our effort on the ellipsoidal particles. We checked the sensitivity of our integrated multi-planar configurations (i.e. IE-1 and IE-2) for non-spherical particle detection. While doing so, we introduced a ratio that accounts for the non-spherical particle electrical signal maximum value with respect to the spherical shape particle electrical signature peak value due to 
the scaling factor. We define the scaling factor as a gradual change in spherical shape, mathematically;

$$
S=b / a
$$

where, $\mathrm{a}$, and $\mathrm{b}$ are the axes lengths of a particle in $\mathrm{x}$ and $\mathrm{y}$-axis directions. For $\mathrm{a}$ sphere $\mathrm{a}=\mathrm{b}$ and $\mathrm{S}=1$. As one of these axes' length changes, the spherical particle transforms into a non-spherical particle. This is illustrated in Supplementary Fig. S8. The scaling factor, S is set to 1 for an exact spherical shape $(\mathrm{a}=\mathrm{b})$ and subsequently varied between 0.1 to 1.5 for a given particle size with an increment of 0.1 as illustrated in a schematic diagram Fig. S8. In Eq. 2, S approaches to unity for a spherical shape. As $\mathrm{S}>1$, particle is enlarged in the vertical direction, however, for $\mathrm{S}<1$, particle shrinks in vertical axis and enlarge in horizontal axis. The scale factor not only takes into consideration the perturbation of the electrical field between microelectrodes by the presence of the particle of various dimensions in the sensing region but also morphology changes in the particle shape. To test our system, we flowed particles $(S=0.1$ to 1.5$)$ through the microchannel in IE-1 and IE-2 designs. The computational results are given in Fig. $4 \mathbf{a}$ and Fig. $\mathbf{4 b}$ for both electrical signatures, $\Delta \mathrm{E}$ and $\left|\mathrm{E}_{2}\right|$ exhibiting a unique footprint for non-spherical shape particles in contrast to spherical particle $(\mathrm{S}=1)$. There is a gradual signal variation manifested along the change in scaling factor, which corresponds to non-spherical shape detection. Notably there exists a symmetry between scaling factor and electrical signal peak values displayed in bar graphs in Fig. 4c and Fig. 4d, which also show the corresponding SNR values. A bar graph displayed both signal maximum value against particle shape ratio (i.e. scaling factor) varied from 0.1 to 1.5 . A percentage difference is computed with respect to exact spherical shape $(S=1)$ to better account for signal sensitivity. As scaling factor increases or decreases the percentage of signal peak shifted accordingly, confirming sensitivity of electrical signature as shape changes slightly from spherical 
to non-spherical. Similarly, the computational results for IE-2 design are given in Supplementary

Fig. S9 for both electrical signatures, $\Delta \mathrm{E}$ and $\left|\mathrm{E}_{2}\right|$.
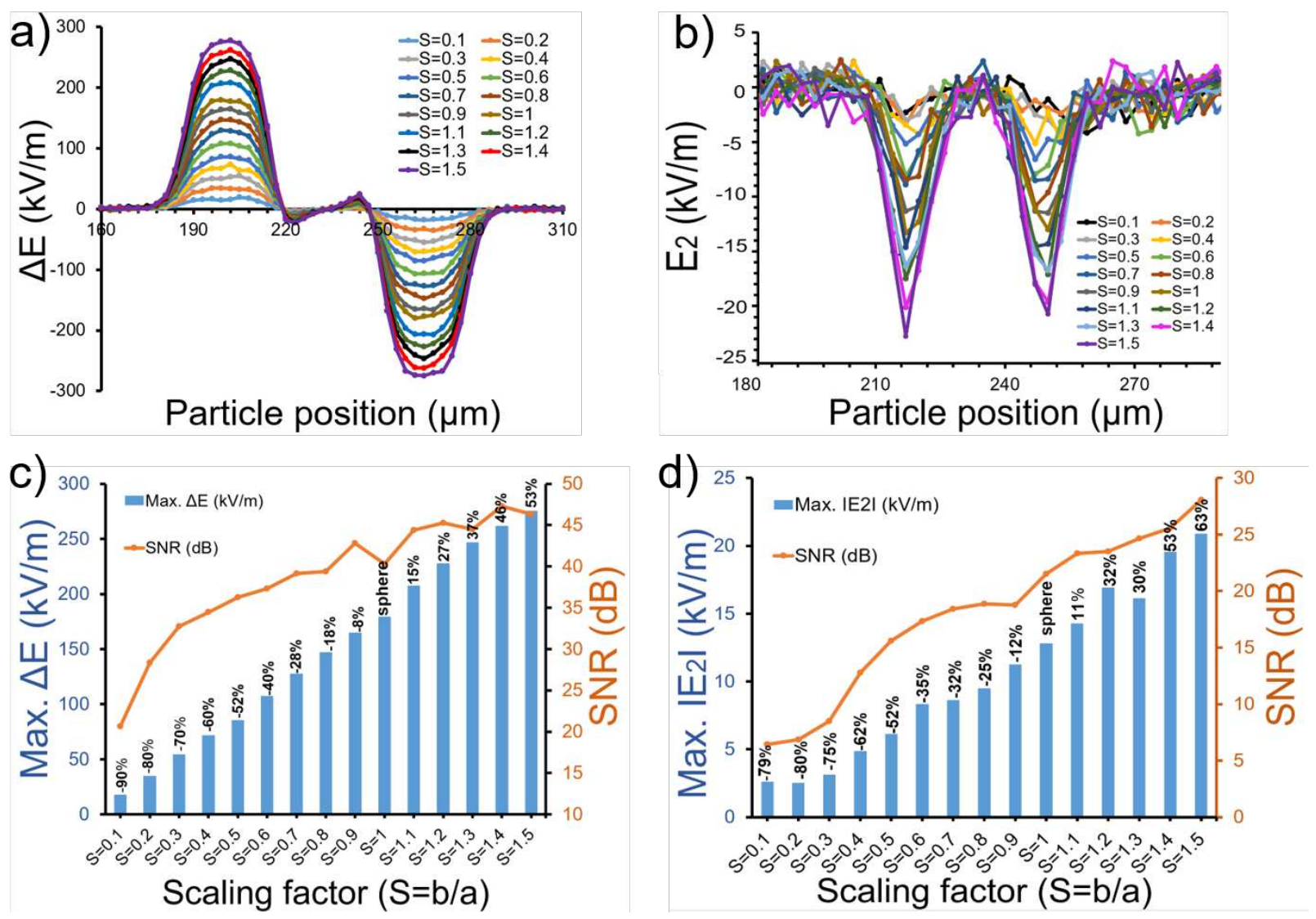

Figure 4. IE-1: Gradual change from spherical to non-spherical shape bioparticle i.e. $\mathrm{S}=0.1-1.5$ (with $\mathrm{a}=5 \mu \mathrm{m}$ remains fixed) for IE-1 design. $(\mathrm{a}, \mathrm{b})$ Simulation of $\Delta \mathrm{E}$ and $\left|\mathrm{E}_{2}\right|$ signals for particle axis ratio, $S=b / a$ from 0.1 to 1.5 . (c, d) Comparison of maximum values of $\Delta E$ and $\left|E_{2}\right|$ and their resctive $\mathrm{SNR}$ in $\mathrm{dB}$ for particle shape ratio, $\mathrm{S}$ from 0.1 to 1.5 .

In further analysis, a ratio of $\Delta \mathrm{E}$ signal peak values of non-spherical particle to spherical particle is computed for IE-1 design. Notably there is a linear correlation between scaling factor and signal ratio which us displayed in bar graphs in Fig. 5. A similar correlation can be observed for the ratio of $\left|E_{2}\right|$ signal peak values of spherical particle and non-spherical particle, represented by bar plots 
in Fig. 5. A similar analysis for IE-2 design configuration is shown in Supplementary Figure S10.

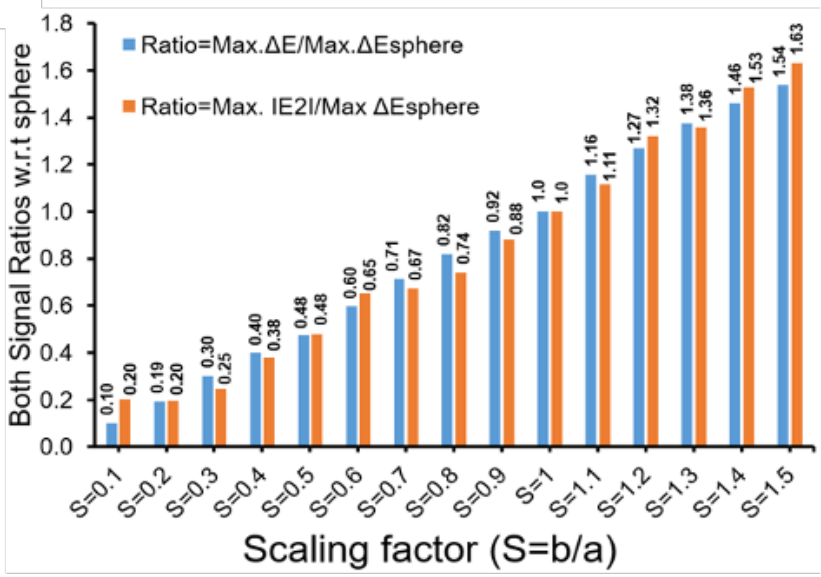

Figure 5. Maximum of signal ratios $\left(\Delta E\right.$ and $\left.\left|E_{2}\right|\right)$ with respect to sphere $(S=b / a=1)$ illustrates a gradual change from spherical to non-spherical shape particle. Scaling factor $(S=b / a)$ increases from 0.1 to 1.5 .

\section{Effect of particle orientation in the micro-channel}

Particle orientation in the microchannel is an important consideration since it will impact the particle position in the electric field and resulting electrical signatures. To test the dependence of our IE-1 design on the particle orientation, we compared the simulation results for the particles orientated at 45-degree angle with respect to x-axis (i.e. representing maximum effect of orientation). The particles were flowed through the microfluidic channel and $\Delta \mathrm{E}$ and $\left|\mathrm{E}_{2}\right|$ were found. Electrical signals were obatined for particle positioned at two different orientation $\left(0^{\circ}\right.$ and $45^{\circ}$ ) within microchannel. $\Delta \mathrm{E}$ and $\left|\mathrm{E}_{2}\right|$ signatures for IE-1 configuration for both particle orientations are shown in Supplementary Figure S11. Comparison of both orientations for IE-1 configuration is given in Fig. 6. Maximum of $\Delta \mathrm{E}$ with respect to scaling factor at both different orientations of particle position is shown in Fig. 6a, while Fig. 6b shows the computed ratios of $\Delta \mathrm{E}$ signal peak values of non-spherical particle ( $\mathrm{S}$ is varied from 0.1 to 1.5 ) to spherical particle at 
both orientations. Comparative analysis shows a small difference because of varied particle orientations in the microchannel. Similarly, maximum of $\left|\mathrm{E}_{2}\right|$ signal with respect to scaling factor at both different orientations of particle position is shown in Fig. 6c, while Fig. 6d shows the computed ratios of $\left|E_{2}\right|$ signal peak values of non-spherical particle to spherical particle at both orientations. We also performed a similar orientation analysis for IE-2 design. $\Delta \mathrm{E}$ and $\left|\mathrm{E}_{2}\right|$ signatures for IE-2 configuration for both particle orientations are shown in Supplementary Fig. S12. Similarly, comparison of $\max \Delta \mathrm{E}$ and $\left|\mathrm{E}_{2}\right|$ with scaling factor for both orientations is shown in Supplementary Fig. S13(a, c). However, comparison of $\Delta \mathrm{E}$ and $\left|\mathrm{E}_{2}\right|$ ratios of non-spherical to spherical particle for both orientations is shown in Supplementary Fig. S13(b, d). Both IE-1 and IE-2 designs were found be independent of orientations of the particle flowing in the microchannel.
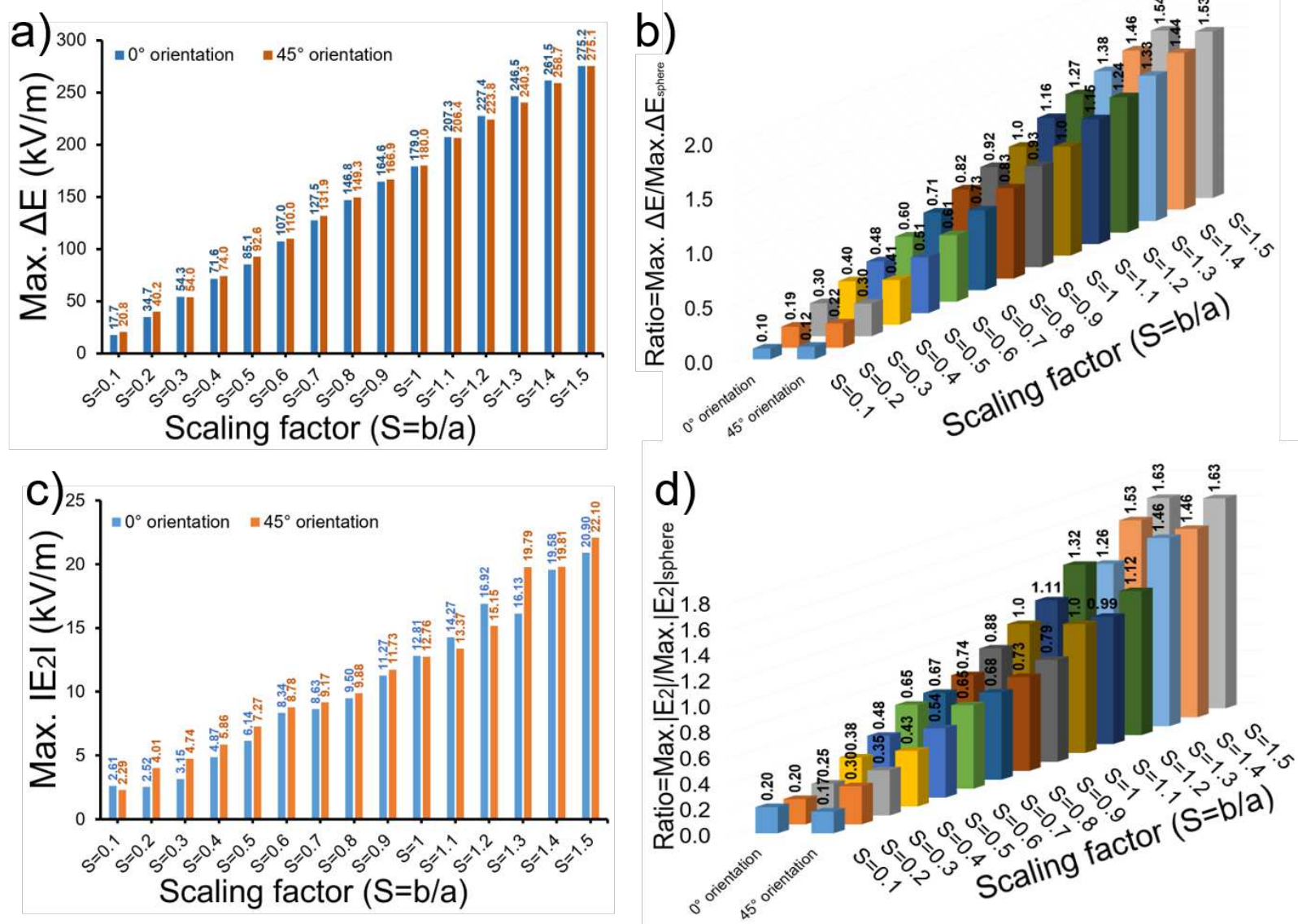
Figure 6. Comparison of spherical and non-spherical particle signal variation with respect to orientation (0-degree and 45-degree) within integrated design IE-1. a) Maximum of $\Delta \mathrm{E}$ with respect to scaling factor at two different orientations of particle position, b) Comparison of ration of maximum value of $\Delta \mathrm{E}$ of non-spherical particle ( $\mathrm{S}=0.1$ to 1.5 ) to spherical particle for both orientations, $c \& d$ ) Comparsion of maximum $\left|E_{2}\right|$ signal and $\left|E_{2}\right|$ ratio at different sacling factors for both orientations.

\section{Relationship of mesh size and noise in the simulations}

Our COMSOL model can be described by partial differential equations (PDE) and the solution to the mathematical model is approximated through the finite element method (FEM) in COMSOL Multiphysics software. FEM is used to discretize the problem and divide the model geometry into tiny elements. This leads to different levels of noise and can be improved by mesh adaptation. By selecting high density mesh distribution, we can decrease errors in physical results and yield more accurate results. While building a mesh, several factors should be taken into consideration, which can be addressed in various features functionality available in the software. This include choosing a mesh sequence type either by an automated process or a customized meshing by manual settings. We have chosen an automated physics-controlled meshing sequence which offers different mesh settings with the options of 'normal', 'fine', 'finer', 'extra fine', and 'extremely fine'. Other lower resolution mesh options are also included e.g., 'coarse', however, in microstructures, these settings give unsatisfactory results with much higher noise.

To investigate the relationship between noise and mesh size, we have simulated a $10 \mu \mathrm{m}$ spherical particle using our integrated design (IE-1) with four mesh settings in order to highlight meshing effects on signal strength and signal to noise ratio (SNR). We have added the corresponding Supplementary Figure S14 and Table S7 and Table S8 with all meshing parameters listed. Although 'extremely fine' mesh setting provide higher element quality, time and space complexity becomes much higher for it to be used. Both 'finer' and 'extra fine' mesh settings give good electric 
signal strengths and signal to noise ratios as compared to 'fine' mesh setting. Supplementary Figure S14c, shows the peak-to-peak simulation noise at these three mesh settings and its effect on overall signal to noise ratio is shown in Supplementary Figure S14b. It should be noted that once a particular mesh size is selected, the noise of the electrical signals defined earlier depends on the electrode designs and configurations.

\section{Differentiation of particles with fixed volumes}

In the next simulation study, we kept the volume of different particles' approximately the same with each other, and adjusted their geometrical parameters. We simulated the spherical, and elliptical particle in horizontal and vertical orientations as shown in Supplementary Figure 15. We flowed these particles thorugh our counter and the results are tabulated in Supplementary Table S9. We observed that the particles with the same width (i.e., sphere and ellipse-x), the pulse width half maximum (FWHM) and pulse amplitude can be used to discriminate elliptical and spherical particle. Both signals $\Delta \mathrm{E} \&\left|\mathrm{E}_{2}\right|$ confirms the particle shape differentiation along with their size. $\Delta \mathrm{E}$ can be used for differentaiting elliptical particle of same volume as spherical one has higher amplitude while smaller peak value for $\left|\mathrm{E}_{2}\right|$ signal differntiates the elliptical shape. When the particles' volume is same and shape is different, we propose an ' $R$ ' value defined as the ratio of PWHM and maximum value of $\Delta \mathrm{E}$ obatined. Two different $\mathrm{R}$ values are calculated based on $\Delta \mathrm{E}$ and $\left|\mathrm{E}_{2}\right|$ signatures i.e., (i) $\mathrm{R}_{\mathrm{A}}=\mathrm{FWHM}(\Delta \mathrm{E}) / \max (\Delta \mathrm{E})$, and (ii) $\mathrm{R}_{\mathrm{B}}=\mathrm{FWHM}(\mathrm{E} 2) / \max (|\mathrm{E} 2|)$. $R_{B}$ is significantly different for elliptical particle as compared to spherical one. $R$ value can be used as a discriminative parameter with pulse width or amplitude. Now, considering a case when the particles length were approximately the same (i.e., sphere and ellipse-y), the pulse amplitudes $\Delta \mathrm{E}$ and $\mathrm{R}_{\mathrm{A}}$ parameters can easily be used to discriminate the particle shape. In comparsion, we found that for fixed volume particle differentiation, $\left|\mathrm{E}_{2}\right|$ signal is less discriminative compared to 
$\Delta \mathrm{E}$ due to non-uniform electric field distribution in coplanar electrodes geometry. Hence an integrated design which provides both electric signals simultaneously helps in better shape differentiation.

In comparison, similar analysis and $\mathrm{R}$ value can also be used to differentiate particles if the particles' volume is not kept same. To further investigate this, we kept length of the particles' $r$ fixed i.e., $10 \mu \mathrm{m}$ and changes the other parameter to vary the volume and shape. The width to length ratio is varied from a lower value to a maximum one, $\mathrm{S}=0.1$ to 1.5 , which changes the volume of the particle along with particles shape, which gradually chnages from elliptical to spherical. We flowed these particles thorugh our counter and the results are tabulated in Supplementary Table 10. We observed that the particles with the same length have almost same pulse width half maximum (FWHM) value, however, the pulse amplitude or the R value can be used to discriminate particle shape (ellipse or sphere). Both signals $\Delta \mathrm{E}$ and $\left|\mathrm{E}_{2}\right|$ confirms the particle shape differentiation as the width of the particle changes. $\Delta \mathrm{E}$ and $\left|\mathrm{E}_{2}\right|$ for elliptical particles of same length but smaller widths compared to spherical, has smaller pulse amplitudes. Further, with different shape and volume particles, we calculated $R$ value. We found that both $R_{A}$ and $R_{B}$ are significantly different when the particles volume and shape is different. Importantly, when the particles' length are approximately the same the impedance pulse amplitude, $\Delta \mathrm{E}$, and $\mathrm{R}_{\mathrm{A}}$ can be used to discriminate the particle shape. Therefore, an integrated design proposed in this study provides a good approximation for particle shape differentiation along with particle size.

\section{Discussion and Conclusion}

In a two-electrode system when a voltage is applied at one electrode surface and ground the opposite electrode, it generates the resulting electric field distribution between them. When a 
particle passes through the sensing zone, it disrupts the electric field distribution, which is recorded as change in electric field value and data is acquired from the electrode surface. Addition of a third middle electrode to the single pair is used for a differential sensing, which provides common mode error reductions to improve signal to noise ratio (SNR). Practically in real world situation this change in electric field will be reflected as the change in impedance which can easily be acquired via Wheatstone bridge i.e., by measuring the potential drop across a resistor, $\mathrm{R}$ as shown in Figure S16b. Figures S16 a \& c further clarifies input and output obtained from electrodes' surface in a 3-electrode layout and in our modified integrated multiplanar electrode configuration (IE-1). Furthermore, the bipolar pulse obtained from the differential signal $\Delta \mathrm{E}$ or $\Delta \mathrm{V}=\mathrm{V}_{1}-\mathrm{V}_{2}$, represents footprints of a particle or a cell which can further be translated into current or amplified as required.

Our proposed sensor design presents a unique direction to differentiate the morphology of the particles flowing in the microchannels. This computational study has demonstrated that integrating microelectrodes in coplanar configuration with top-bottom electrode configuration will increase the sensor's sensitivity and SNR. It results in the effective electrical differentiation of the particles' size and morphology. We demonstrated non-spherical bioparticle discrimination by using our proposed integrated multi-planar configuration of microelectrodes (IE) owing to their unique changes in the electrical signature. The separation is accomplished using a uniform electric field which was generated by an integrated geometry of a microelectrodes. Moreover, sensor sensitivity is further investigated by simulating spherical and elliptical shaped particles. A microsensor consisting of a constricted microchannel and a modified microelectrode configuration is proposed and evaluated in order to measure the transformation of a single spherical shape bioparticle into elliptical by analyzing the changes in the electric field intensity. A strong correlation was observed between the peak amplitude of signals ( $\Delta \mathrm{E}$ and $\left.\left|\mathrm{E}_{2}\right|\right)$ of non-spherical (i.e., elliptical) particle axis 
ratio (i.e., scaling factor) to peak amplitude of signal for a spherical particle. Two different integrated designs i.e., IE-1 and IE-2 were proposed. Both designs provided good signal strength, SNR value and were able to differentiate spherical vs. non spherical particles. In comparison, IE1 design produced better results because of lower limit of detection. However, it should be noted that the limit of detection and the sensor sensitivity can be further improved by employed postprocessing data filtering techniques to remove noise. Furthermore, reducing the channel height or increasing the input electric field strength will further improve the accuracy in differentiation smaller particles.

Particle rotation while flowing in the microfluidic channels is an important consideration since it will impact the particle position in the electric field and resulting electrical signatures. In various practical experimental conditions e.g., microparticles of elliptical shape (such as E. coli) won't always align themselves parallel to the sensing zone while flowing through the microchannel. Nonspherical microparticles may cross the sensing zone in different orientations e.g., vertical or diagonal positions. Such examples have been reported earlier in many cases ${ }^{44-50}$. The variation in the orientation for particle flow depends on many factors including flow rate, pressure, biasing, dilution factor, hydrodynamic forces and other manual sample handling e.g., 3D asymmetrical inlet holes in microfluidic devices. Therefor we try to accommodate two most possible orientations i.e., $0^{0}$ and $45^{\circ}$ that particles can take while flowing through the channel as an in-depth computational study. So, we evaluated the dependence on the particle orientation in the microchannel at $0^{0}$ and $45^{\circ}$ positions along the horizontal axis. Both IE-1 and IE-2 designs showed small variations in the corresponding electrical signatures. Collection of multiple electrical signatures $\Delta \mathrm{E}$ and $\left|\mathrm{E}_{2}\right|$ can be used to develop multi-variate computational models based on machine learning techniques to improve the sensor accuracy ${ }^{43}$. Table S11 details a comparative 
analysis of our study with earlier reports on the similar topic. We compared the specific features including size, shape and cell classification in single cell cytometry in the Table S11 and list the limitations. Our integrated design has been proven to be more effective in the classification of nonspherical bioparticles compared to existing techniques by using the ellipsoidal shape as proof-ofconcepts for the potential differentiation of the various non-spherical biological entities, for instance blood cells, bacteria and others in future.

\section{Conflicts of interest}

The authors declare no conflicts of interest.

\section{Acknowledgements}

This work was financially supported by the Department of Electrical and Computer Engineering, Rutgers the state university of New Jersey, USA. Authors also acknowledge the funding support from Rutgers Global Health Institute and Rutgers Global - Collaborative Research Grant. A.F. was partly supported by the International Research Support Initiative Program (IRSIP) of Pakistan Higher Education Commission. 


\section{References}

1. K. Asami, Journal of Non-Crystalline Solids, 2002, 305, 268-277.

2. Y. Katsumoto, K. Tatsumi, T. Doi and K. Nakabe, International Journal of Heat and Fluid Flow, 2010, 31, 985-995.

3. M.-H. Wang and L.-S. Jang, Biosensors and bioelectronics, 2009, 24, 2830-2835.

4. D. Zhang, H. Bi, B. Liu and L. Qiao, ACS Publications, 2018.

5. R. F. Kushner, P. De Vries and R. Gudivaka, The American journal of clinical nutrition, 1996, 64, 503S-509S.

6. A. Vembadi, A. Menachery and M. A. Qasaimeh, Frontiers in Bioengineering and Biotechnology, 2019, 7.

7. S. M. A. Iqbal and N. Z. Butt, SN Applied Sciences, 2019, 1, 1290.

8. U. Hassan and R. Bashir, Biomedical microdevices, 2014, 16, 697-704.

9. U. Hassan, N. N. Watkins, B. Reddy, G. Damhorst and R. Bashir, Nature protocols, 2016, 11, 714726.

10. N. N. Watkins, U. Hassan, G. Damhorst, H. Ni, A. Vaid, W. Rodriguez and R. Bashir, Science translational medicine, 2013, 5, 214ra170-214ra170.

11. K. Kiilerich-Pedersen and N. Rozlosnik, Diagnostics, 2012, 2, 83-96.

12. B. G. Andryukov, N. N. Besednova, R. V. Romashko, T. S. Zaporozhets and T. A. Efimov, Biosensors, 2020, 10, 11.

13. J. Chen, C. Xue, Y. Zhao, D. Chen, M.-H. Wu and J. Wang, International journal of molecular sciences, 2015, 16, 9804-9830.

14. S. Park, R. R. Ang, S. P. Duffy, J. Bazov, K. N. Chi, P. C. Black and H. Ma, PloS one, 2014, 9, e85264.

15. M. Hosokawa, H. Kenmotsu, Y. Koh, T. Yoshino, T. Yoshikawa, T. Naito, T. Takahashi, H. Murakami, Y. Nakamura and A. Tsuya, PloS one, 2013, 8, e67466.

16. M. Islam, H. Brink, S. Blanche, C. DiPrete, T. Bongiorno, N. Stone, A. Liu, A. Philip, G. Wang and W. Lam, Scientific reports, 2017, 7, 1-12.

17. T. Ye, N. Phan-Thien, B. C. Khoo and C. T. Lim, Biophysical journal, 2013, 105, 1103-1109.

18. D. R. Gossett, H. T. Tse, S. Lee, A. T. Clark and D. Di Carlo, 14th International Conference on Miniaturized System for Chemistry and Life Sciences Oct, 2010.

19. M. Shaker, L. Colella, F. Caselli, P. Bisegna and P. Renaud, Lab on a Chip, 2014, 14, 2548-2555.

20. D. C. Rees, T. N. Williams and M. T. Gladwin, The Lancet, 2010, 376, 2018-2031. 
21. M. Kotb, H. Ramadan, R. Shams El Din, H. Motaweh and E. El-Bassiouni, European Scientific Journal June, 2014, 10, 365-378.

22. A. F. Cowman and B. S. Crabb, Cell, 2006, 124, 755-766.

23. J. Cottet, A. Kehren, H. Van Lintel, F. Buret, M. Frénéa-Robin and P. Renaud, Microfluidics and Nanofluidics, 2019, 23, 11.

24. F. A. Coumans, G. van Dalum, M. Beck and L. W. Terstappen, PloS one, 2013, 8, e61770.

25. P. Bankó, S. Y. Lee, V. Nagygyörgy, M. Zrínyi, C. H. Chae, D. H. Cho and A. Telekes, Journal of hematology \& oncology, 2019, 12, 48.

26. B. Shashni, S. Ariyasu, R. Takeda, T. Suzuki, S. Shiina, K. Akimoto, T. Maeda, N. Aikawa, R. Abe and T. Osaki, Biological and Pharmaceutical Bulletin, 2018, 41, 487-503.

27. M. Simeonova, D. Wachner and J. Gimsa, Bioelectrochemistry, 2002, 56, 215-218.

28. X. Xie, Z. Zhang, X. Ge, X. Zhao, L. Hao, Z. Cheng, W. Zhou, Y. Du, L. Wang and F. Tian, Analytical chemistry, 2019, 91, 13398-13406.

29. C. Honrado, L. Ciuffreda, D. Spencer, L. Ranford-Cartwright and H. Morgan, Journal of The Royal Society Interface, 2018, 15, 20180416.

30. K. K. Zeming, S. Ranjan and Y. Zhang, Nature communications, 2013, 4, 1-8.

31. S. Hu, Y. Zhao and X.-I. Qian, Journal of Electrostatics, 2015, 75, 72-76.

32. B. Brazey, J. Cottet, A. Bolopion, H. Van Lintel, P. Renaud and M. Gauthier, Lab on a Chip, 2018, $18,818-831$.

33. R. Rodriguez-Trujillo, M. A. Ajine, A. Orzan, M. D. Mar, F. Larsen, C. H. Clausen and W. E. Svendsen, Sensors and Actuators B: Chemical, 2014, 190, 922-927.

34. R. Rodriguez-Trujillo, O. Castillo-Fernandez, M. Garrido, M. Arundell, A. Valencia and G. Gomila, Biosensors and Bioelectronics, 2008, 24, 290-296.

35. J. Mok, M. N. Mindrinos, R. W. Davis and M. Javanmard, Proceedings of the National Academy of Sciences, 2014, 111, 2110-2115.

36. D. Holmes, D. Pettigrew, C. H. Reccius, J. D. Gwyer, C. van Berkel, J. Holloway, D. E. Davies and H. Morgan, Lab on a Chip, 2009, 9, 2881-2889.

37. F. Caselli and P. Bisegna, Medical Engineering \& Physics, 2017, 48, 81-89.

38. V. Errico, A. De Ninno, F. R. Bertani, L. Businaro, P. Bisegna and F. Caselli, Sensors and Actuators B: Chemical, 2017, 247, 580-586.

39. J. Hong, D. S. Yoon, S. K. Kim, T. S. Kim, S. Kim, E. Y. Pak and K. No, Lab on a Chip, 2005, 5, 270-279.

40. C. H. Clausen, G. E. Skands, C. V. Bertelsen and W. E. Svendsen, Micromachines, 2015, 6, 110-120. 
41. A. De Ninno, R. Reale, A. Giovinazzo, F. R. Bertani, L. Businaro, P. Bisegna, C. Matteucci and F. Caselli, Biosensors and Bioelectronics, 2020, 150, 111887.

42. C. H. Clausen, M. Dimaki, C. V. Bertelsen, G. E. Skands, R. Rodriguez-Trujillo, J. D. Thomsen and W. E. Svendsen, Sensors, 2018, 18, 3496.

43. U. Hassan, R. Zhu and R. Bashir, Lab on a Chip, 2018, 18, 1231-1240.

44. J.-W. Choi, S. Rosset, M. Niklaus, J. R. Adleman, H. Shea and D. Psaltis, Lab on a Chip, 2010, 10, 783-788.

45. Z. Zhang, T. E. Kimkes and M. Heinemann, Scientific reports, 2019, 9, 1-9.

46. F. Si, B. Li, W. Margolin and S. X. Sun, Scientific reports, 2015, 5, 11367.

47. A. J. Mathijssen, N. Figueroa-Morales, G. Junot, É. Clément, A. Lindner and A. Zöttl, Nature communications, 2019, 10, 1-12.

48. J. Männik, F. Wu, F. J. Hol, P. Bisicchia, D. J. Sherratt, J. E. Keymer and C. Dekker, Proceedings of the National Academy of Sciences, 2012, 109, 6957-6962.

49. G. Holzner, S. Stavrakis and A. DeMello, Analytical chemistry, 2017, 89, 11653-11663.

50. D. I. Cattoni, J.-B. Fiche, A. Valeri, T. Mignot and M. Nöllmann, PloS one, 2013, 8, e76268. 


\section{Figures}
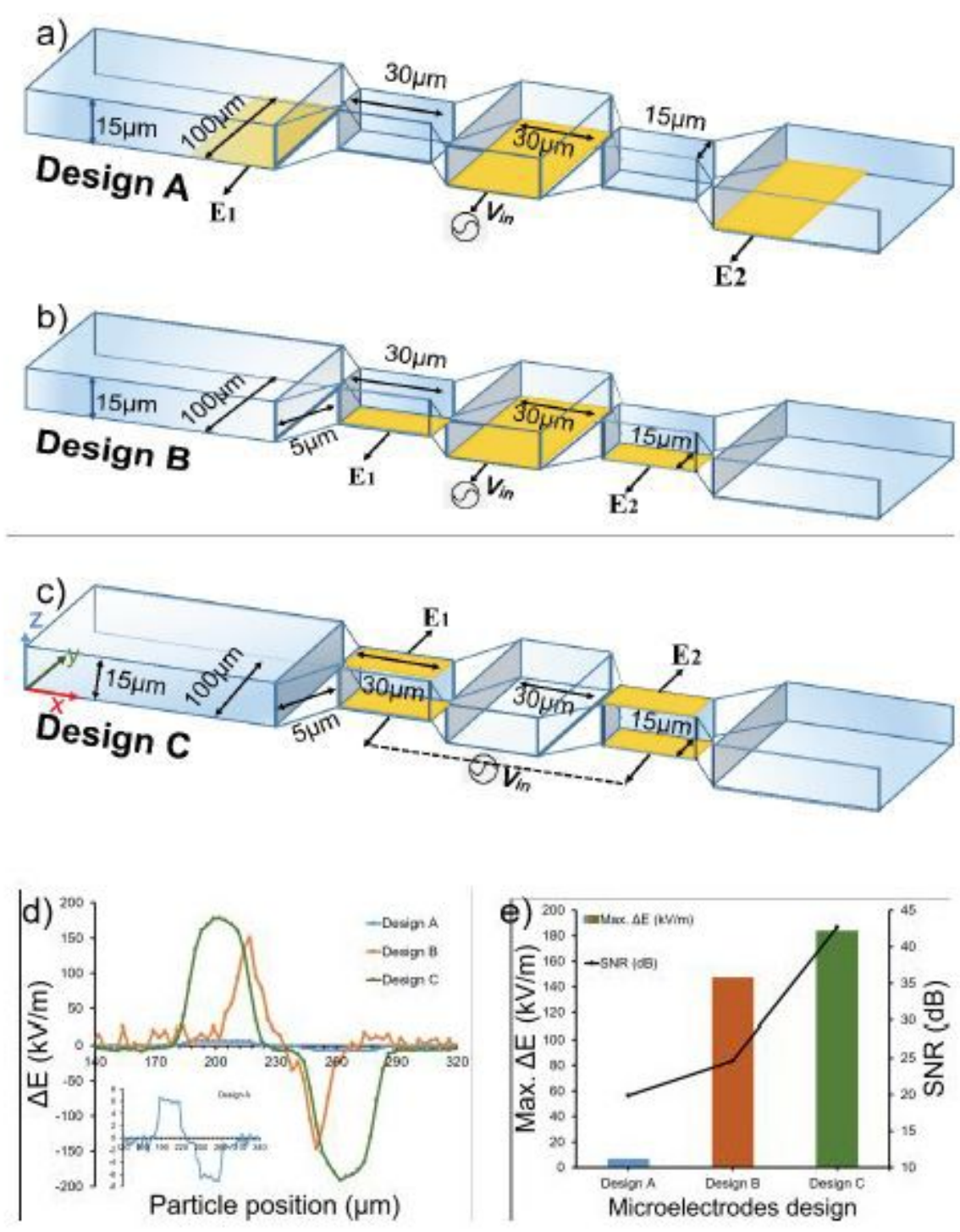

\section{Figure 1}

3-D view of all microfluidic channels with microelectrodes designs with geometrical dimensions, a) Coplanar microelectrodes layout (Design A), b) Modified coplanar microelectrode layout (Design B) with electrodes in narrow aperture, c) Top bottom microelectrodes layout (Design C), d \& e) Electrical signal 
comparison in three microelectrode layouts. Design B, C outperformed Design A in signal strength. However, Design $C$ produces highest signal strength and lowest noise.
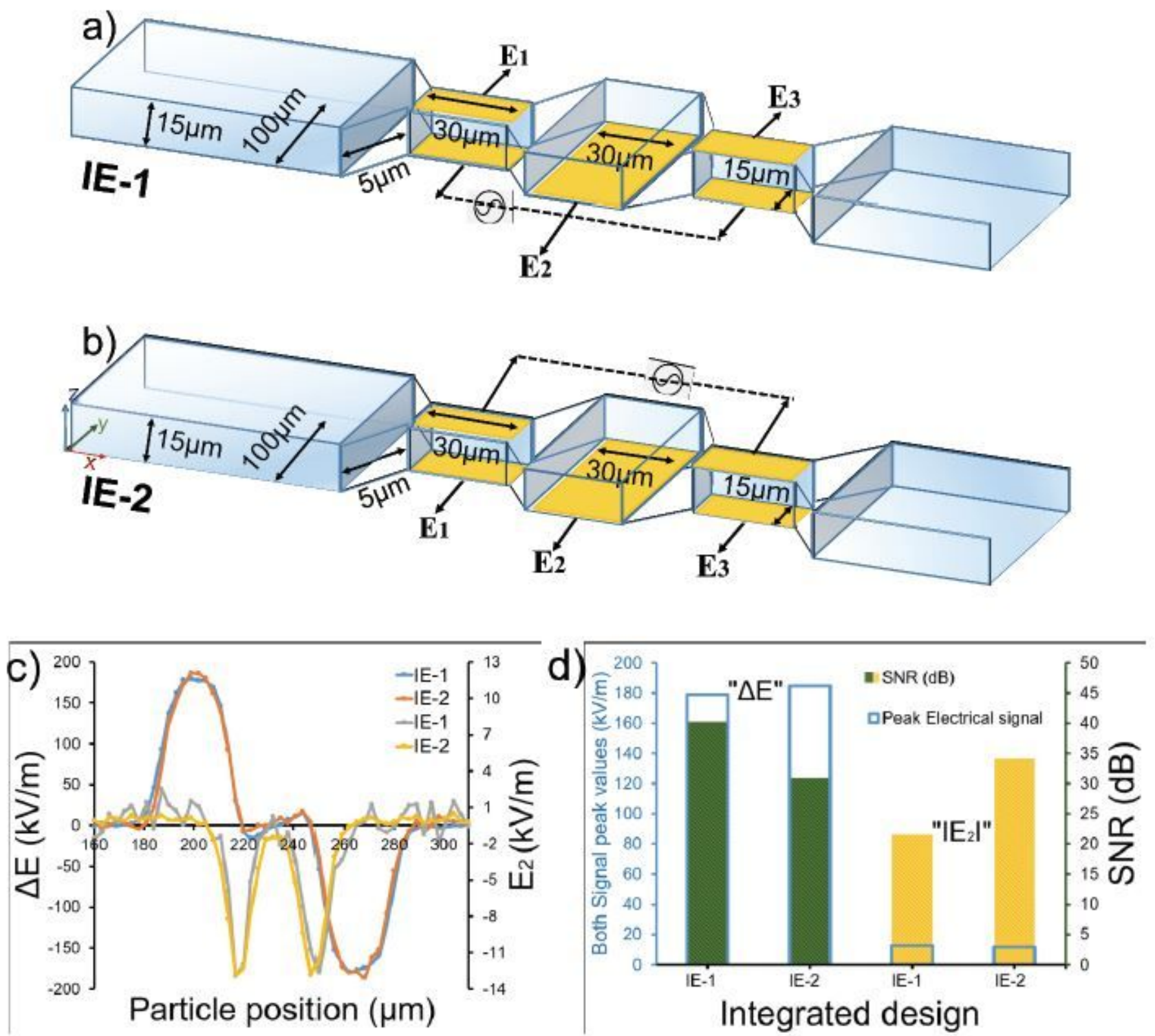

Figure 2

Detailed geometrical dimensions for integrated multi-planar designs of microelectrodes (IE-1 and IE-2) with microfluidic channel in 3-D view, a \& b) Integrated electrode designs with input signal and output signals; IE-1 (a) and IE-2 (b), c) Output signals $\triangle E=E 1-E 3$ (bipolar pulse) and E2 (unipolar pulse) from IE-1 and IE-2 relative to the particle position in the channel, d) Comparison between the $\Delta E=E 1-E 3$ and $E 2$ signal peak values with respect to SNR in $\mathrm{dB}$ for IE-1 and IE-2. 

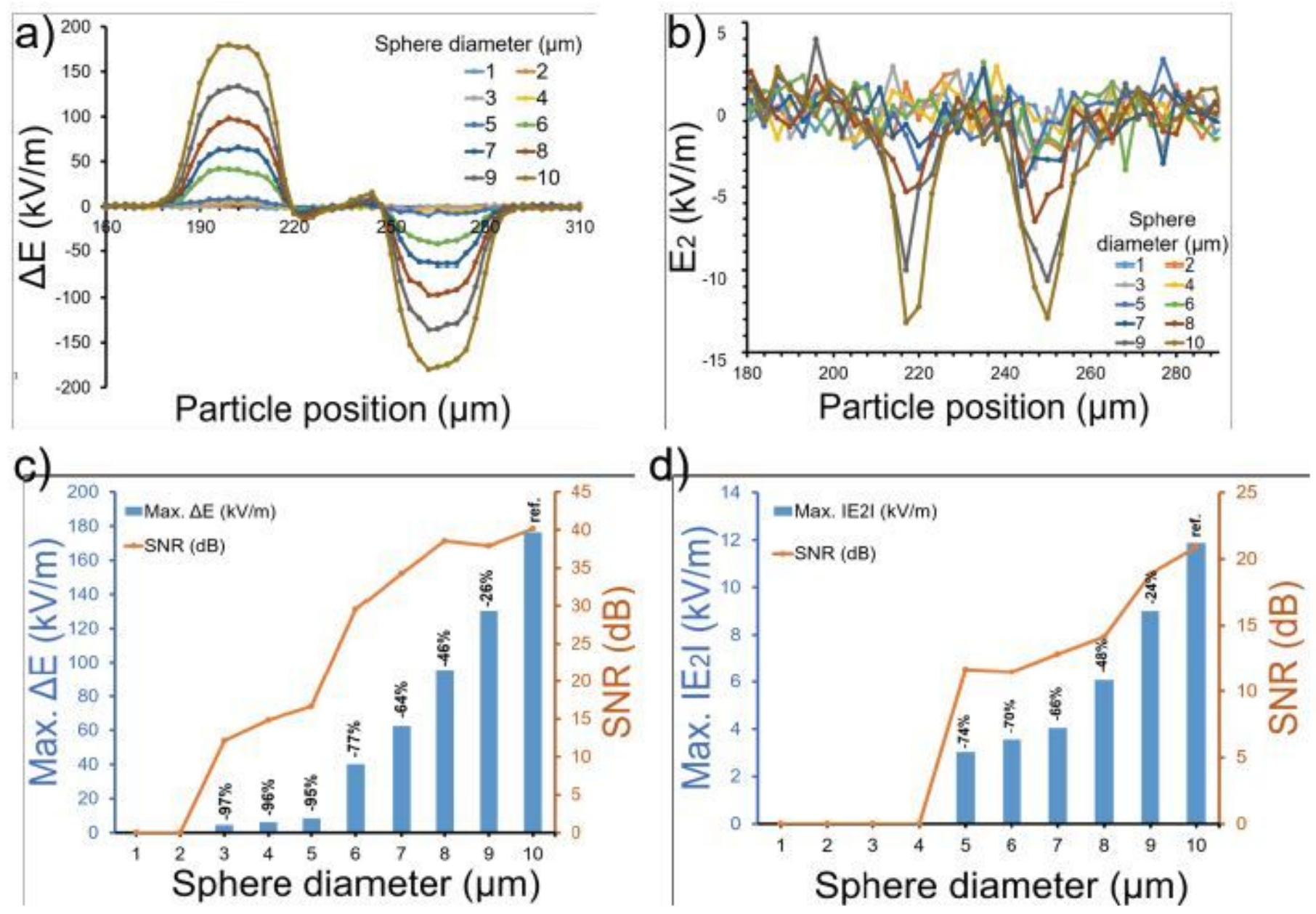

Figure 3

a \& b) Simulation of $\Delta E$ and $E 2$ signals for spherical particle versus diameter increment from 1 to 10 in $\mu \mathrm{m}, \mathrm{c} \& \mathrm{~d}$ ) Comparison of maximum values of $\Delta \mathrm{E}$ and $\mathbb{E}$ 2『and their resctive SNR in $\mathrm{dB}$ for spherical particle diameter ranges from 1 to $10 \mu \mathrm{m}$. 

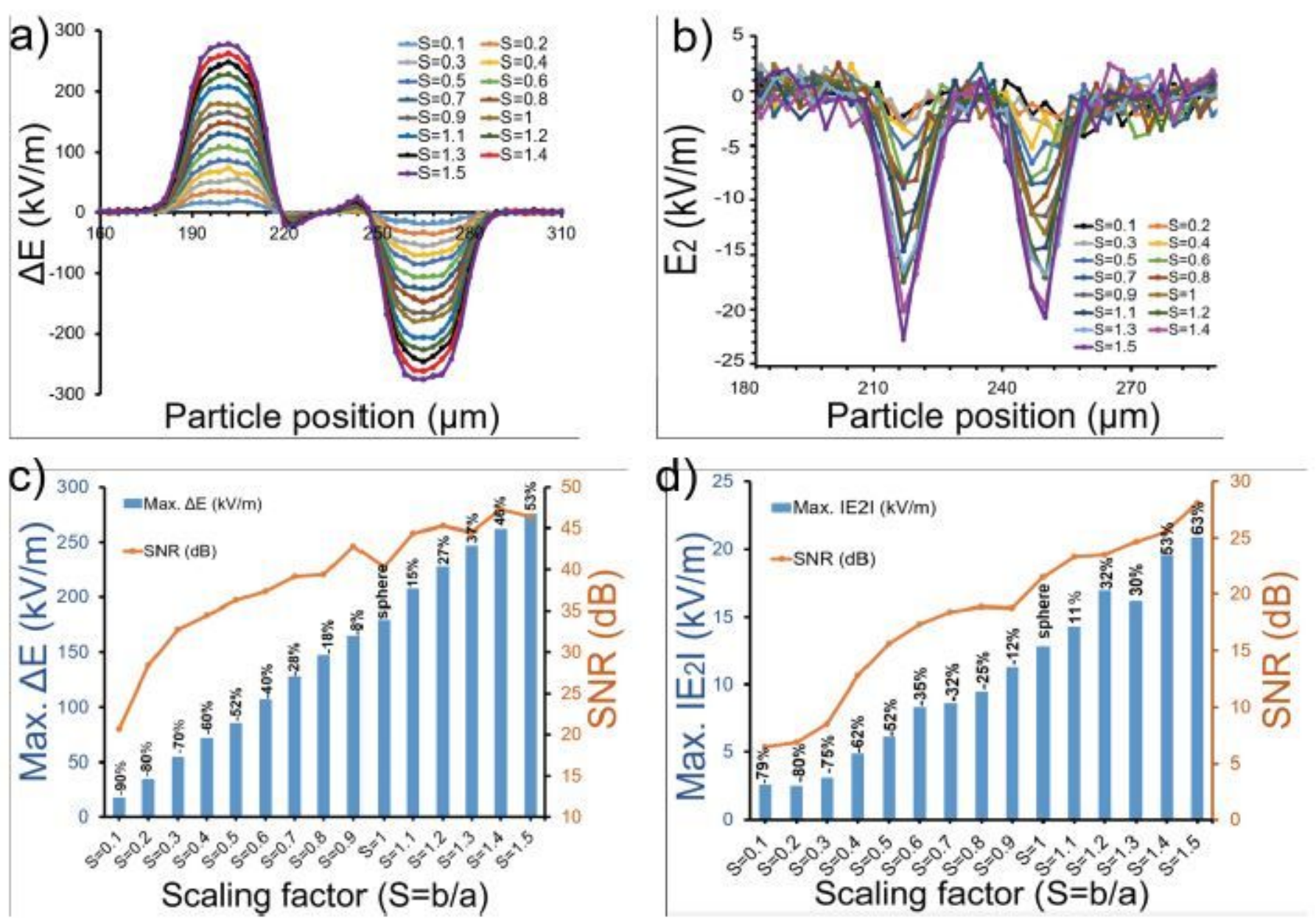

Figure 4

IE-1: Gradual change from spherical to non-spherical shape bioparticle i.e. $S=0.1-1.5$ (with a=5 $\mu \mathrm{m}$ remains fixed) for $\mathrm{IE}-1$ design. $(\mathrm{a}, \mathrm{b})$ Simulation of $\Delta \mathrm{E}$ and $\otimes \mathrm{E} 2$ \signals for particle axis ratio, $\mathrm{S}=\mathrm{b} / \mathrm{a}$ from 0.1 to 1.5. (c, d) Comparison of maximum values of $\Delta \mathrm{E}$ and $\triangle \mathrm{E} 2$ \and their resctive SNR in $\mathrm{dB}$ for particle shape ratio, $\mathrm{S}$ from 0.1 to 1.5 . 


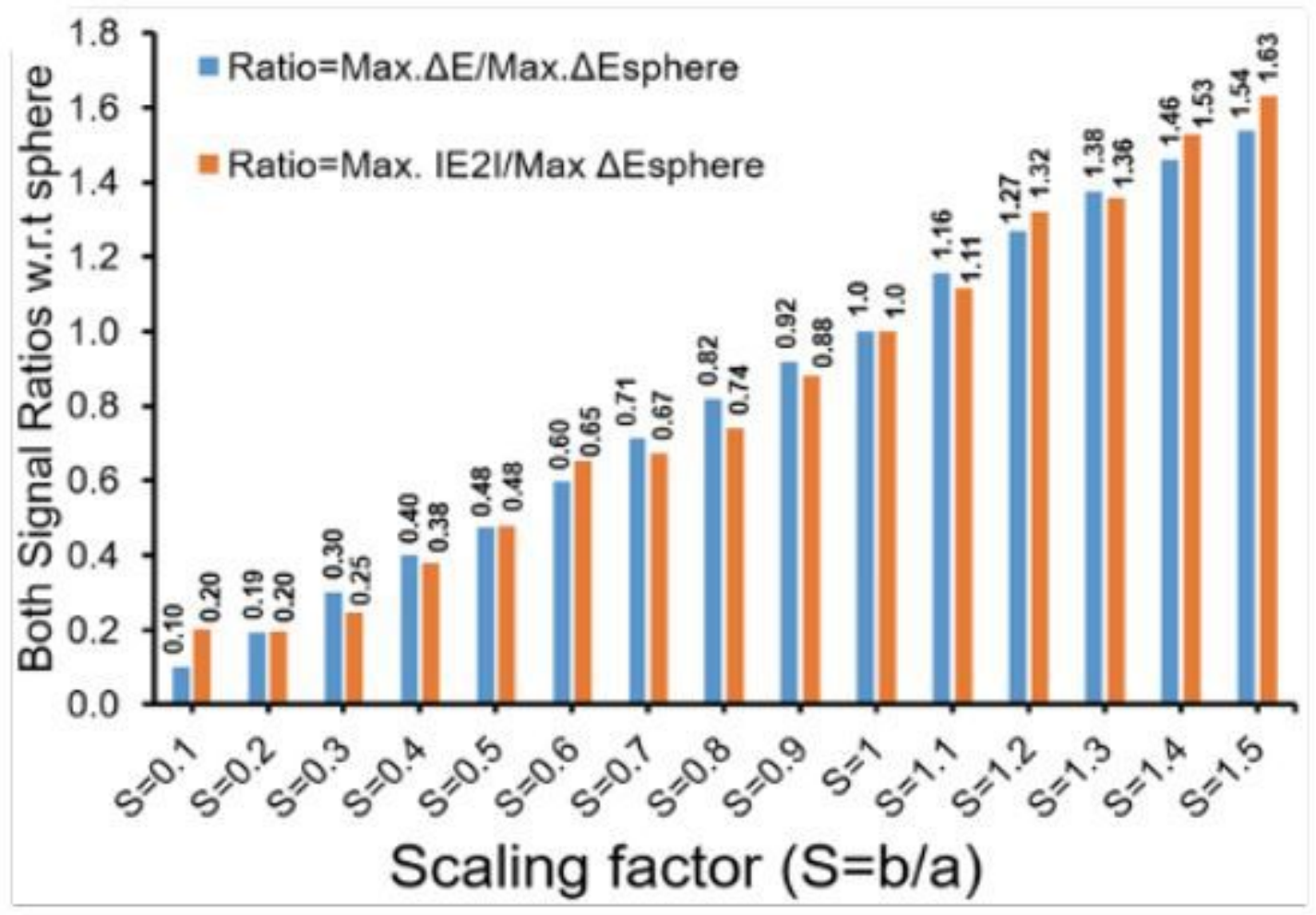

Figure 5

Maximum of signal ratios ( $\Delta E$ and $\triangle E 2 \varangle)$ with respect to sphere $(S=b / a=1)$ illustrates a gradual change from spherical to non-spherical shape particle. Scaling factor $(S=b / a)$ increases from 0.1 to 1.5 . 

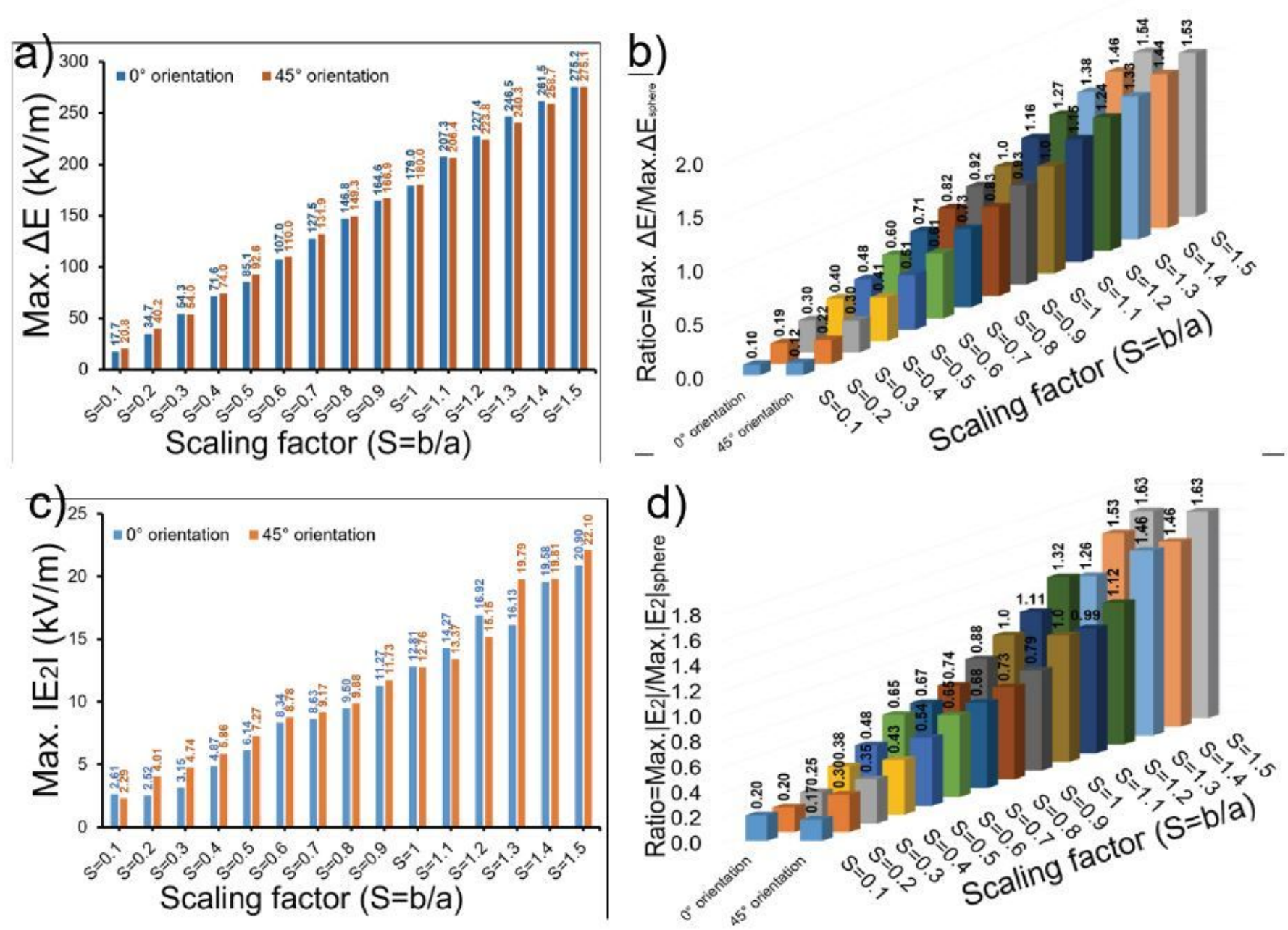

Figure 6

Comparison of spherical and non-spherical particle signal variation with respect to orientation (0-degree and 45-degree) within integrated design IE-1. a) Maximum of $\Delta \mathrm{E}$ with respect to scaling factor at two different orientations of particle position, $b$ ) Comparison of ration of maximum value of $\Delta E$ of nonspherical particle ( $S=0.1$ to 1.5 ) to spherical particle for both orientations, c \& d) Comparsion of maximum |E2| signal and |E2| ratio at different sacling factors for both orientations.

\section{Supplementary Files}

This is a list of supplementary files associated with this preprint. Click to download.

- SIfinal.pdf 